\title{
Forward modeling of volume electron microscopy (vEM) of stained resin-embedded biological samples
}

Yu Yuan ${ }^{1}$, Sabrina Clusiau ${ }^{1}$, Raynald Gauvin ${ }^{2}$, Christopher Bleck ${ }^{3}$, Adrian Phoulady ${ }^{4}$, Pouya Tavousi ${ }^{5}$, Sina Shahbazmohamadi ${ }^{6}$, Nicolas Piché ${ }^{1}$ and Mike Marsh ${ }^{7}$

${ }^{1}$ Object Research Systems, United States, ${ }^{2}$ McGill University, Montreal, Quebec, Canada, ${ }^{3}$ National Heart, Lung, and Blood Institute, Bethesda, Maryland, United States, ${ }^{4}$ REFINE Center, University of Connecticut, United States, ${ }^{5}$ UConn Tech Park, University of Connecticut, storrs, Connecticut, United States, ${ }^{6}$ University of Connecticut, Storrs, Connecticut, United States, ${ }^{7}$ Object Research Systems, Denver, Colorado, United States

Image formation in the scanning electron microscope (SEM) is a complicated process that starts with interaction between the incident electron beam and the sample being imaged. The physics of electron-matter interaction has been described and modeled by MC-Xray in the past, although, only recently, has that modeling been integrated into a user-friendly software framework Dragonfly and accelerated and extended from 0dimensional point simulations to 2D simulations that mimic the 2D rastering of the e-beam in an SEM [1]. Understanding the image formation and forward-modeling of images in the SEM offers two benefits. First, knowing a priori how variations in microscope conditions affect contrast and resolution permits a microscopist to optimize imaging parameters in advance. Second, having a realistic phantom paired with the corresponding forward-modeled images equips machine learning researchers with suitable ground truth for training deep learning and other artificial intelligence models for automatic segmentation of the microstructure observed in new micrographs. We show here image simulation of 2D micrographs from a simple 3D phantom structure of heavy-metal stained resin-embedded biological tissue and demonstrate that the simulated images strongly resemble the experimental micrographs, both qualitatively and quantitatively.

The forward modeling requires a phantom, or digital structure, of known geometry and material composition. In this work, we derive a 3-phase phantom from SEM images of murine rod internal segments, prepared by high-pressure freezing, freeze-substitution with osmium tetroxide, followed by durcupan resin embedding at room temperature. This sample was selected because it demonstrates some of the structural complexity of stained biological samples, but the image histogram suggests that the material composition is rather simple. We infer only three major phases: stained membranes at the highest backscattered electron (BSE) signal, with a lower BSE signal for the intracellular and intra-organelle areas, and an even lower BSE signal for the extracellular space (see Figure 1A). A stack of 2D images was collected by FIB-SEM serial sectioning on a Zeiss Crossbeam, using the energy-selective backscatter (EsB) detector. The stack of images was segmented into 3 phases, plasma membrane and organelle membranes, intracellular, and extracellular resin.

The input conditions for the simulation include the total count and the landing voltage of the incident electrons at every point along the surface of the $3 \mathrm{D}$ phantom. We match the experimental landing voltage $(1.5 \mathrm{keV})$ and use a similar fluence of electrons. Because our simulation results encode the energy of the backscattered electrons, we simulate the experimental EsB by choosing not to accumulate in our image any of the electrons whose energy is below a filter threshold $(1.0 \mathrm{keV})$.

The remaining free parameter of the simulation is the elemental weight-fraction for each of the phases encoded in our 3D phantom. The precise elemental weight-fraction in biological samples like these is difficult to determine experimentally. For our simulations, we visually observed image contrast and noise similar to the experimental images when we used osmium weight-fraction approximating $20 \%$. To further refine the quality of our simulation, we varied two parameters: 1) the weight-fraction of osmium from $10 \%$ to $40 \%$, and 2) the noise level in the phantom. That noise level allows us to encode a non-uniform osmium binding throughout the three material phases. We find that $20 \%$ weight-fraction osmium is qualitatively similar to the experimental 
micrographs, while early quantitative assessments suggest that $10 \%$ weight-fraction osmium may be most similar. We note that the simulated micrograph exhibits structural details from the subsurface, due to the interaction volume which exceeds the slice thickness of our model; this emphasizes that such images must be simulated, as they cannot be derived simply by spatial convolution of the phantom with naive noise models.

We expect that the accuracy of the simulation can be enhanced by improving the geometry of phantom, such as constraining membranes to have thicknesses consistent with known measurements. And, we can further constrain the heavy metal weight-fraction to better match experimental observations [2]. Ultimately, this simulation capability can be paired with an upscaled phantom generation capacity from technology such as generative adversarial networks (GANs) to provide abundant paired phantoms and simulated images for machine learning.
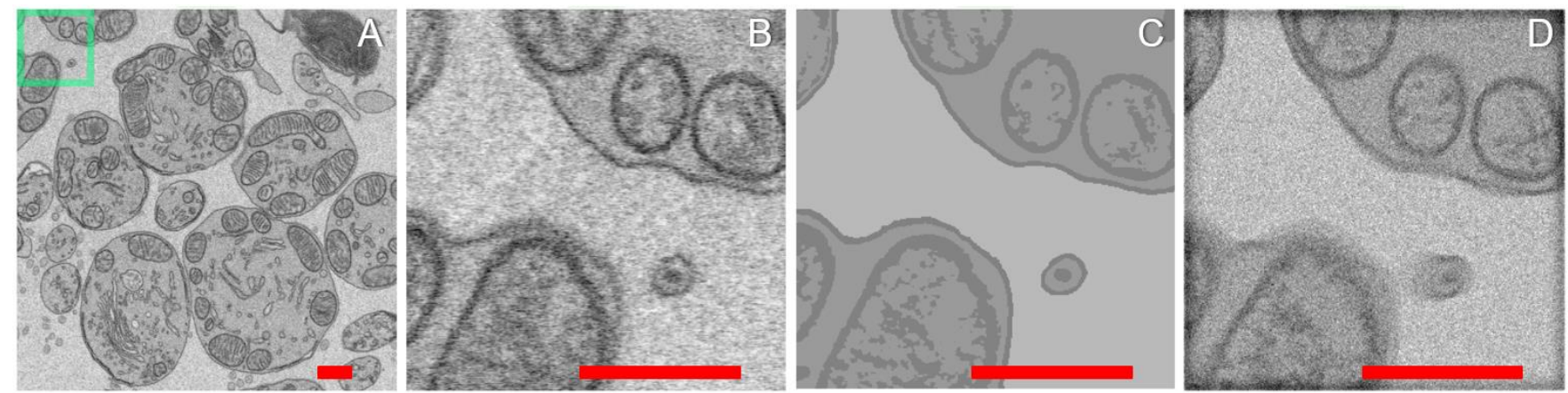

Figure 1. (a) Energy-filtered micrograph collected on Zeiss Crossbeam. (b) Enlargement of the greenhighlighted region of the micrograph shown in the previous panel. (c) A single slice from the 3-phase 3D phantom. (d) Micrograph simulated for the 3D phantom, computed with imaging and filter conditions comparable to the actual micrograph, shown in b. Red scale bars are $500 \mathrm{~nm}$.

\section{References}

[1] S Rudinsky et al., Microscopy and Microanalysis 25(S2) (2019), p. 222-223.

[2] A Fera et al., Journal of Microscopy 277(2) (2020), p. 71-78. 